\title{
RESISTIVITY LOGGING IN A MULTISCALE ISOTROPIC POROUS MEDIUM WITH LOG-NORMAL DISTRIBUTED CONDUCTIVITY
}

\author{
E.P. Kurochkina ${ }^{a}$, O.N. Soboleva ${ }^{b}$, M.I. Epov ${ }^{c}$ \\ ${ }^{a}$ Institute of Thermophysics, SB RAS, Novosibirsk, Russia \\ ${ }^{b}$ Institute of Computational Mathematics and Mathematical Geophysics, SB RAS, Novosibirsk, Russia \\ ${ }^{c}$ Institute of Petroleum Geology and Geophysics, SB RAS, Novosibirsk, Russia \\ The effective coefficients of a conductivity field for estimating an average value of the current \\ density and its variance for the problem of resistivity logging in a multiscale isotropic porous medium \\ are considered. A conductivity field has pulsations from an extremely wide range of scales and log- \\ normal statistics. For the modelling of the conductivity we use Kolmogorov's multiplicative cascades. \\ The problem is solved by the method of subgrid modelling. The results are verified by the 3D numerical \\ modelling. \\ Рассматриваются эффективные коэффициенты поля проводимости для оценки среднего зна- \\ чения плотности тока и его изменений для задачи электрического каротажа в мультимасштабной \\ изотропной пористой среде. Поле проводимости характеризуется пульсациями в крайне широком \\ диапазоне масштабов и логнормальной статистикой. Для моделирования проводимости использу- \\ ются мультипликативные каскады Колмогорова. Задача решается методом подсеточного модели- \\ рования. Результаты контролируются 3D-моделированием.
}

PACS: $02.70 . \mathrm{Rr} ; 61.43 . \mathrm{Gt}$

\section{INTRODUCTION}

Natural porous media are essentially inhomogeneous. Large-scale (coarse) elements of a medium structure, such as, easily recognizable layers or intercalations are directly described by the model. Small-scale details of conductivity, porosity, permeability are unknown. They should be considered within the statistical approach, introducing effective parameters. To take into account the intermittency of an inhomogeneous medium one can use the ideas proposed by Kolmogorov [1]. In this approach, the basic properties of media are hierarchical spatial structurization and power dependencies. 


\section{MAIN EQUATIONS AND A CONDUCTIVITY MODEL}

We consider the problem of the direct-current in the isotropic inhomogeneous medium [2]:

$$
\begin{gathered}
\nabla_{i} \sigma(\mathbf{x}) \nabla_{\mathbf{i}} \mathbf{U}(\mathbf{x})=\mathbf{0},\left.\quad \mathbf{U}(\mathbf{x})\right|_{\mathbf{s}}=\mathbf{U}_{\mathbf{0}}(\mathbf{x}), \\
\mathbf{E}(\mathbf{x})=-\nabla \mathbf{U}(\mathbf{x}), \quad \mathbf{j}(\mathbf{x})=\sigma(\mathbf{x}) \mathbf{E}(\mathbf{x}),
\end{gathered}
$$

where $\sigma(\mathbf{x})$ is conductivity of the medium; $U(\mathbf{x}), \mathbf{E}(\mathbf{x})$ are potential and electrical fields; $\mathbf{j}(\mathbf{x})$ is density of the current. We suppose that the conductivity is constant outside the volume $V$ with a boundary $S$. Let the field of conductivity be known. This means that it is measured in a volume of a small size $l_{0}$ for each point $\mathbf{x}$. A random function of the spatial coordinates $\sigma(\mathbf{x})$ is considered as limit of the conductivity $\sigma(\mathbf{x})_{l_{0}}$. As $l_{0} \rightarrow 0$, we have $\sigma(\mathbf{x})_{l_{0}} \rightarrow \sigma(\mathbf{x})$. To pass to a coarser grid $l_{1}$, one can smooth the resultant field $\sigma(\mathbf{x})_{l_{1}}$ using the scale $l_{1}>l_{0}$. Similar to [1,3], we consider a dimensionless function $\psi\left(\mathbf{x}, l, l_{1}\right)=\sigma(\mathbf{x})_{l} / \sigma(\mathbf{x})_{1}$, where $\sigma(\mathbf{x})_{l_{1}}$ is the conductivity $\sigma(\mathbf{x})_{l_{0}}$ smoothed over the scale $l_{1}$. The field $\psi\left(\mathbf{x}, l, l_{1}\right)$ has too many arguments. We define a simpler field that contains the same information. We expand $\psi\left(\mathbf{x}, l_{1}, l_{3}\right)$ and $\psi\left(\mathbf{x}, l_{2}, l_{3}\right)$ in series at $l_{2}$. Rejecting the terms of second order and using the evident equality $\psi\left(\mathbf{x}, l_{1}, l_{3}\right)=\psi\left(\mathbf{x}, l_{1}, l_{2}\right) \psi\left(\mathbf{x}, l_{2}, l_{3}\right)$, we obtain

$$
\frac{\partial \psi\left(\mathbf{x}, l_{1}, l_{2}\right)}{\partial l_{2}}=\frac{1}{l_{2}} \psi\left(\mathbf{x}, l_{1}, l_{2}\right) \varphi\left(\mathbf{x}, l_{2}\right),
$$

where $\varphi\left(\mathbf{x}, l_{2}\right)=\left.\left(\partial \psi\left(\mathbf{x}, l_{2}, \lambda l_{2}\right) / \partial \lambda\right)\right|_{\lambda=1}, \lambda=l_{3} / l_{2}$. From (2) it follows:

$$
\frac{\partial \ln \sigma(\mathbf{x}, l)}{\partial \ln l}=\varphi(\mathbf{x}, l) .
$$

The solution to Eq. (3) has the form

$$
\sigma(\mathbf{x}) l_{0}=\sigma_{0} \exp \left[-\int_{l_{0}}^{L} \varphi\left(\mathbf{x}, l_{1}\right) \frac{d l_{1}}{l_{1}}\right],
$$

where $\sigma_{0}$ is constant. We suppose that the conductivity has heterogeneities of the scale $l$ from the interval $\left(l_{0}, L\right)$, where $l_{0}$ is minimal and $L$ is maximal scales of measurements $L^{3} \ll V, \sigma(\mathbf{x})=\sigma(\mathbf{x})_{l_{0}}$. The field $\varphi(\mathbf{x}, l)$ is assumed to be homogeneous and isotropic, then a correlation function is

$$
\left\langle\varphi(\mathbf{x}, l) \varphi\left(\mathbf{y}, l_{1}\right)\right\rangle-\langle\varphi(\mathbf{x}, l)\rangle\left\langle\varphi\left(\mathbf{y}, l_{1}\right)\right\rangle=\Phi\left((\mathbf{x}-\mathbf{y})^{2}, l, l_{1}\right),
$$

where \langle\rangle is probability averaging. If the function $\varphi$ is statistically invariant to the scale transform, its correlation is satisfied by $\Phi\left(\frac{(\mathbf{x}-\mathbf{y})^{2}}{l^{2}}, \frac{l_{1}}{l}\right)$. The discrete approximation of the fields $\varphi\left(\mathbf{x}, l_{1}\right)$ will be considered to render probabilistic models. In this approximation, the fields $\varphi(\mathbf{x}, l), \varphi\left(\mathbf{y}, l_{1}\right)$ with different scales $l, l_{1}$ at any $\mathbf{x}, \mathbf{y}$ are considered to be statistically independent. This is usually assumed in the scaling models and reflects the decay of statistical dependence, when the scales of fluctuations become different magnitude. The latter was 
proposed in [1]. To describe the probability distribution for the integral from (4) for large $L / l$, we use the theorem about sums of independent variables. If the variance of $\varphi(\mathbf{x}, l)$ at a given point exists, then the theorem says that the factor $\int_{l_{0}}^{L} \varphi\left(\mathbf{x}, l_{1}\right) \frac{d l_{1}}{l_{1}}$ for very large $L / l_{0}$ tends to a normal field. In the opposite case (the second correlation function does not exist), the integral over $d l / l$ tends to a field described by a stable distribution. The case of a stable distribution is considered in [4]. In our work, it is assumed that at $l_{0}<l<L \varphi(\mathbf{x}, l)$ has normal distribution.

\section{SUBGRID MODEL}

The conductivity function $\sigma(\mathbf{x})=\sigma(\mathbf{x})_{l_{0}}$ is divided into two components with respect to the scale $l$. The large-scale component $\sigma(\mathbf{x}, l)$ is obtained by statistical averaging over all $\varphi\left(\mathbf{x}, l_{1}\right)$ with $l_{0}<l_{1}<l, l-l_{0}=d l$, where $d l$ is small. A small-scale (subgrid) component is equal to $\sigma^{\prime}(\mathbf{x})=\sigma(\mathbf{x})-\sigma(\mathbf{x}, l)$ :

$$
\begin{aligned}
\sigma(\mathbf{x}, l)=\sigma_{0} \exp \left[-\int_{l}^{L} \varphi\left(\mathbf{x}, l_{1}\right) \frac{d l_{1}}{l_{1}}\right]\left\langle\exp \left[-\int_{l_{0}}^{l} \varphi\left(\mathbf{x}, l_{1}\right) \frac{d l_{1}}{l_{1}}\right]\right\rangle, \\
\sigma^{\prime}(\mathbf{x})=\sigma(\mathbf{x}, l)\left[\frac{\exp \left[-\int_{l_{0}}^{l} \varphi\left(\mathbf{x}, l_{1}\right) \frac{d l_{1}}{l_{1}}\right]}{\left\langle\exp \left[-\int_{l_{0}}^{l} \varphi\left(\mathbf{x}, l_{1}\right) \frac{d l_{1}}{l_{1}}\right]\right\rangle}-1\right] .
\end{aligned}
$$

A large-scale (ongrid) component of the potential $U(\mathbf{x}, l)$ is obtained as averaging Eq. (1) in which a large-scale component of conductivity is fixed and a small component $\sigma^{\prime}(\mathbf{x})$ is a random variable $U(\mathbf{x}, l)=\langle U(\mathbf{x})\rangle_{\sigma(\mathbf{x}, l)}$. A subgrid component of the potential is $U^{\prime}(\mathbf{x})=U(\mathbf{x})-U(\mathbf{x}, l)$. Substituting the expression for $U(\mathbf{x}), \sigma(\mathbf{x})$ in Eq. (1) and averaging over the small-scale component, we obtain

$$
\nabla_{i}\left[\sigma(\mathbf{x}, l) \nabla_{i} U(\mathbf{x}, l)+\left\langle\sigma^{\prime}(\mathbf{x}) \nabla_{i} U^{\prime}(\mathbf{x})\right\rangle_{\sigma(\mathbf{x}, l)}\right]=0,
$$

where \langle\rangle$_{\sigma(\mathbf{x}, l)}$ denotes statistical averaging over $l_{1}<l$, when $\sigma(\mathbf{x}, l)$ is fixed. The second term in Eq. (8) is unknown. It cannot be rejected without preliminary estimation, since the correlation between the conductivity and the potential gradient may be substantial. The choice of the form of the second term in (8) determines the subgrid model. This expression is estimated using perturbation theory. Let the scale $l$ be close to the smallest scale $l_{0}$. Subtracting (8) from (1) and ignoring terms of the second order of smallness, we obtain the subgrid equation for the potential $U^{\prime}(\mathbf{x})$ :

$$
\Delta U^{\prime}(\mathbf{x})=-\frac{1}{\sigma(\mathbf{x}, l)} \nabla_{i} \sigma^{\prime}(\mathbf{x}) \nabla_{i} U(\mathbf{x}, l) .
$$


The variables $\sigma(\mathbf{x}, l), U(\mathbf{x}, l)$ in the right-hand side of Eq. (9) are considered known, their derivatives varying slower than $\sigma^{\prime}(\mathbf{x}), U^{\prime}(\mathbf{x})$ and their derivatives. Therefore,

$$
U^{\prime}(\mathbf{x})=\frac{1}{4 \pi \sigma(\mathbf{x}, l)} \int_{V} \frac{1}{r} \nabla_{j}^{\prime} \sigma^{\prime}\left(\mathbf{x}^{\prime}\right) d \mathbf{x}^{\prime} \nabla_{j} U(\mathbf{x}, l),
$$

where $r=\left|\mathbf{x}-\mathbf{x}^{\prime}\right|$. From (6), (10), using the equality $\int \frac{x_{j} x_{m}}{r^{2} d \vartheta}=\frac{4 \pi \delta_{j m}}{3}$, where $\vartheta$ is a full solid angle, we obtain

$$
\left\langle\sigma(\mathbf{x}) \nabla_{i} U(\mathbf{x})\right\rangle_{\sigma(\mathbf{x})_{l}} \simeq\left[1+\left(\frac{1}{6} \Phi_{0}(l)-\langle\varphi\rangle\right) \frac{d l}{l}\right] \sigma(\mathbf{x})_{l} \nabla_{i} U(\mathbf{x}, l),
$$

where $\Phi_{0}(l)=\Phi(0, l)$. Here, the integration over the finite volume $V$ is replaced by the integration with infinite limits, because the correlation function $\Phi$ is small outside the domain of scale $L$. Such a substitution gives a coarse estimation near to the boundary, but this does not affect the determined mean values, because $L^{3} \ll V$. In the limit $l \rightarrow l_{0}$, we come to the expression for the effective coefficient, which correctly describes a mean value of the current density:

$$
\sigma_{\text {eff }}(\mathbf{x})=\sigma_{0 l}^{1} \exp \left[-\int_{l}^{L} \varphi\left(\mathbf{x}, l_{1}\right) \frac{d l_{1}}{l_{1}}\right]
$$

where

$$
\frac{d \ln \sigma_{0 l}^{1}}{d \ln l}=-\langle\varphi\rangle+\frac{1}{6} \Phi_{0}(l) .
$$

If a function $\varphi$ is statistically invariant to the scale transform, the solution to Eq. (13) has especially a simple form: $\sigma_{0 l}^{1}=\sigma_{0 L}(l / L)^{-\langle\varphi\rangle+\Phi_{0} / 6}$, where the constant $\sigma_{0 L}$ describes the density for the largest scale $\langle\mathbf{j}\rangle=-\sigma_{0 L} \nabla\langle U\rangle$. In the same way, we come to the effective coefficients for the correlation tensor of the current density. The effective coefficients for diagonal components of the tensor are

$$
\frac{d \ln \left(\sigma_{0 l}^{2}\right)^{2}}{d \ln l}=\left(\frac{14}{15} \Phi_{0}(l)-2\langle\varphi\rangle\right), \quad \frac{d \ln \left(\sigma_{0 l}^{3}\right)^{2}}{d \ln l}=\left(\Phi_{0}(l)-2\langle\varphi\rangle\right) .
$$

If a function $\varphi$ is statistically invariant to the scale transform,

$$
\mathbf{w}=\theta_{1} A \mathbf{v}_{L}-\theta_{2} I \mathbf{v}_{L},
$$

where $w_{i}=\left\langle j_{i}^{2}(\mathbf{x})\right\rangle-\left\langle j_{i}(\mathbf{x})\right\rangle^{2}, \quad v_{i L}=j_{i L}^{2}(\mathbf{x}), \quad \theta_{1}=\sigma_{0 L}^{2}\left(l_{0} / L\right)^{\Phi_{0}-2\langle\varphi\rangle} / 3, \quad \theta_{2}=$ $\sigma_{0 L}^{2}\left(l_{0} / L\right)^{\Phi_{0} / 3-2\langle\varphi\rangle}, A$ is a matrix, $a_{i i}=2\left(l_{0} / L\right)^{\Phi_{0} / 15}+1, a_{i j}=-\left(l_{0} / L\right)^{\Phi_{0} / 15}+1$, $I$ is a unit matrix. For non-diagonal components of the tensor, we have

$$
\begin{gathered}
\frac{d \ln \left(\sigma_{0 l}^{4}\right)^{2}}{d \ln l}=\left(\frac{4}{5} \Phi_{0}(l)-2\langle\varphi\rangle\right) . \\
{\left[\left\langle j_{i}(\mathbf{x}) j_{j}(\mathbf{x})\right\rangle-\left\langle j_{i}(\mathbf{x})\right\rangle\left\langle j_{j}(\mathbf{x})\right\rangle\right]_{\mathrm{eff}}=} \\
=\sigma_{0 L}^{2}\left[\left(l_{0} / L\right)^{4 / 5 \Phi_{0}-2\langle\varphi\rangle}-\left(l_{0} / L\right)^{-2\langle\varphi\rangle+\Phi_{0} / 3}\right] j_{i}(\mathbf{x}, L) j_{k}(\mathbf{x}, L) .
\end{gathered}
$$




\section{NUMERICAL MODELLING}

For the numerical calculation we use dimensionless variables. The problem is solved for $\sigma_{0}=1$ in a unit cube with a unit potential difference along $Y$ axis. The integral in (4) is replaced by a finite difference formula, in which it is convenient to pass to a logarithm with base 2 , where $l=2^{\tau}$.

$$
\sigma(\mathbf{x})_{l_{0}}=2\left(-\sum_{i=-5}^{-3} \varphi\left(\mathbf{x}, \tau_{i}\right) \Delta \tau\right)
$$

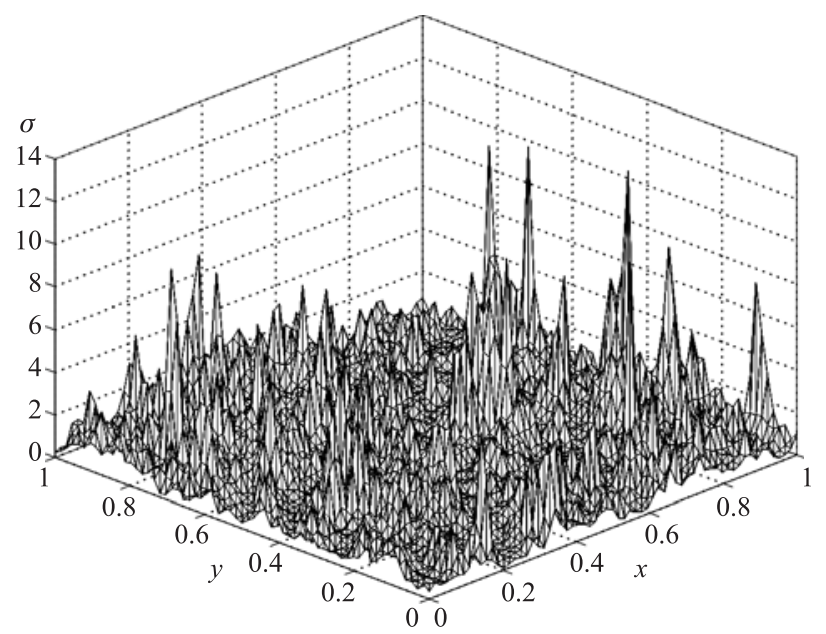

Fig. 1. The self-similar conductivity in the mid-span section $z=1 / 2$ calculated by formula (17) for $\langle\varphi\rangle=0.15$

For the numerical modelling, the scale step $\Delta \tau$ is equal to one, $\tau_{i}=-3,-4,-5$, corresponding to $l=1 / 64,1 / 32,1 / 16$. To calculate $\varphi$, we use the correlation function $\left\langle\varphi\left(\mathbf{x}, \tau_{i}\right) \varphi\left(\mathbf{y}, \tau_{j}\right)\right\rangle-\left\langle\varphi\left(\mathbf{x}, \tau_{i}\right)\right\rangle\left\langle\varphi\left(\mathbf{y}, \tau_{j}\right)\right\rangle=\left(\Phi_{0} / \ln 2\right) \exp \left[-(\mathbf{x}-\mathbf{y})^{2} / 2^{2 \tau_{i}}\right]$. The constants $\Phi_{0},\langle\varphi\rangle$ should be chosen from experimental data for natural media [5]. We choose $\Phi_{0}=0.3$. In Fig. 1, we have self-similar conductivity in the mid-span section for formula (17). The structure of the correlation matrix allows us to represent it in the form of a direct product of four matrices low dimensionality and to apply the algorithm «along rows and columns» for numerical simulation [6]. According to the procedure deriving subgrid formulas, we have to numerically solve the complete problem and perform the probability averaging over

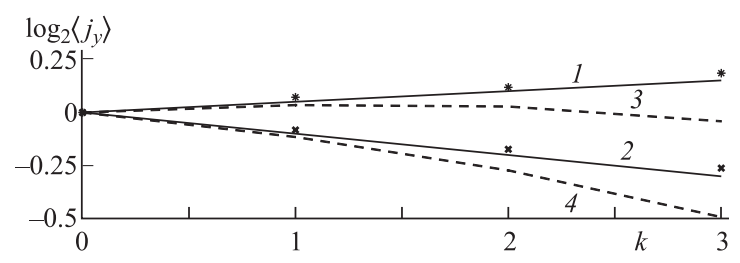

Fig. 2. The dependence of the logarithm of the mean $Y$ component of the current density on the considered number of the scales in the model 
small-scale fluctuations of conductivity to verify the subgrid formulas. As a result, we obtain a subgrid term, which can be compared to theoretical expression. In the present work, a more efficient verification has been carried out. We numerically solve dimensionless Eq. (1) for conductivities using one, two and three summands in the exponent of formula (17). An iterative method combined with Fourier transform and the sweep method is used. Then, we determine corresponding mean values replacing the statistical averaging by spatial averaging and calculate the same mean values using theoretical formulas. We also compare the results obtained to our theoretical formulas with the results obtained with «ordinary» perturbations theory. The ergodic hypothesis is verified. Figure 2 gives: lines 1,2 are $\left\langle\log _{2} j_{y}(\mathbf{x})\right\rangle$ obtained by theoretical formulas (12), (13) for $\langle\varphi\rangle=0$ and $\langle\varphi\rangle=0.15$, respectively; dashed lines 3,4 are the result obtained with «ordinary» perturbation theory; stars and crosslets mark the result of numerical modelling. The mean $X, Z$ components of the current desity are equal to 0 . The result of numerical verification for the variance of the current density components $j_{y}, j_{x}$ is plotted in Fig. 3, where $D_{x}=\log _{2}\left[\left\langle j_{x}^{2}(\mathbf{x})\right\rangle-\left\langle j_{x}(\mathbf{x})\right\rangle^{2}\right], D_{y}=\log _{2}\left[\left\langle j_{y}^{2}(\mathbf{x})\right\rangle-\left\langle j_{y}(\mathbf{x})\right\rangle^{2}\right]$. Dashed lines 1, 2 are the result obtained with «ordinary» perturbation theory; lines 3, 4 are obtained by theoretical formula (15) for $\langle\varphi\rangle=0.15$; stars mark the result of numerical modelling. For the longitudinal component variance of the density $\left(j_{y}(\mathbf{x})\right)$ the numerical result is in good agreement with the estimation obtained with theoretical formulas (14), (15). For the transverse component, the distinction of the two results is in the limits of the calculation error.

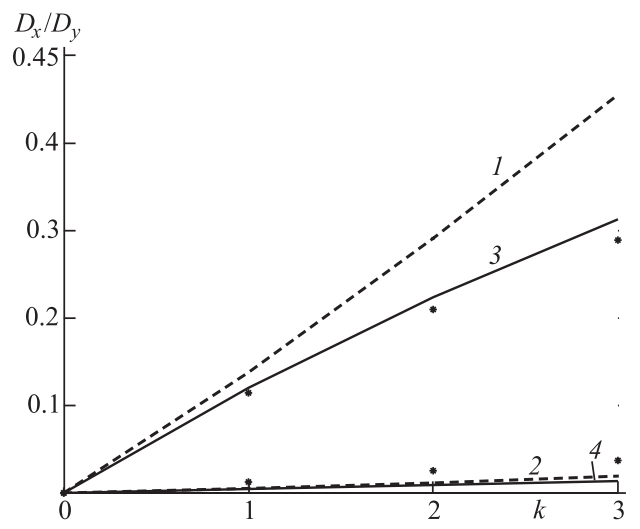

Fig. 3. The dependence of the logarithm variance of the components of the current density $j_{y}, j_{x}$ on the considered number of scales in the model

\section{CONCLUSIONS}

In this work we obtain the formulas including the effect of small-scale fluctuations in the calculation of mean characteristics of the current density. The conductivity field is approximated by the extremely inhomogeneous field close to a multifractal. If $l \rightarrow l_{0}$ in formula (4), we obtain the multifractal. However, this approach is within the apparatus of differential equations and theory of random processes. The main objects of the theory are fields, whose properties can, in principle, be measured. The considered medium has the scale-invariance conductivity. We obtain that the mean value of the current density and 
the pairwise differences, the sum of single-point moments have exponential dependencies on the scale of inhomogeneity. The exponents in the formulas for effective coefficients are determined. The numerical verification is in good agreement with theoretical formulas as well as with numerical results.

The work is supported by Russian Foundation for Basic Research, project No. 04-0564415, No.06-05-64149, Integration project No. 75, SB RAS.

\section{REFERENCES}

1. Kolmogorov A. N. // J. Fluid Mech. 1962. V.13. P. 82.

2. Landau L.D., Lifshitz E.M. Electrodynamics of Continuous Media. Oxford; Elmsford; N. Y.: Pergamon Press, 1984.

3. Kuz'min G. A., Soboleva O.N. // J. Appl. Mech. Tech. Phys. 2002. V.43. P. 583.

4. Soboleva O. N. // J. Appl. Mech. Tech. Phys. 2005. V.46. P. 891.

5. Sahimi M. // Rev. Mod. Phys. 1993. V.65, No.4. P. 1393.

6. Ogorodnikov V.A., Prigarin S.M. Numerical Modeling of Random Processes and Fields: Algorithms and Applications. Utrecht: Kluwer, 1996. 